\title{
The transfer of location-based control requires location-based conflict
}

\author{
Lauren Pickel $^{1}$ • Jay Pratt ${ }^{1}$ • Blaire J. Weidler ${ }^{2}$ \\ Published online: 15 July 2019 \\ (C) The Psychonomic Society, Inc. 2019
}

\begin{abstract}
It is well supported that stimulus-driven control of attention varies depending on the degree of conflict previously encountered in a given location. Previous research has further shown that control settings established in conflict-biased locations can transfer to nearby unbiased items. However, these spatial transfer effects have only been shown using incompatible flanking arrows (i.e., stimuli that trigger spatial information) to elicit conflict in a flanker task. Here we examine the generalizability of transfer of control by examining if it can occur across a range of tasks. We employ a classic Stroop task (Experiment 1), a spatially segregated Stroop task (Experiment 2), and a spatial Stroop task (Experiment 3). Location-specific proportion compatibility effects were observed in all variations of the Stroop task tested; however, transfer to unbiased items occurred only in the spatial Stroop task in Experiment 3. This suggests that the transfer of cognitive control settings within spatial categories may occur only in tasks where the source of conflict is spatial, as arises in tasks with arrow and direction word stimuli.
\end{abstract}

Keywords Cognitive and attentional control $\cdot$ Attention: space-based

\section{Introduction}

"Cognitive control" is a term that encompasses numerous processes that bias attention to optimize performance on a person's current task. This is necessary in many situations in which irrelevant and often conflicting information must be ignored in order to attend to information relevant to current demands (e.g., Cohen, 2017). For example, when driving, cognitive control must be used to allocate appropriate attention to stimuli relevant to the task, such as street signs and the movement of pedestrians, while simultaneously excluding distractions such as billboards.

Cognitive control has traditionally been thought of as an effortful process that can be upregulated in response to particular environmental demands such as a relatively high overall level of conflict (e.g., Lowe \& Mitterer, 1982). Recently, however, it has also been demonstrated that control can be triggered quickly and flexibly by features of a stimulus (e.g., its location) that are not known prior to a stimulus' appearance.

Blaire J. Weidler

blaire.weidler@gmail.com

1 University of Toronto, Toronto, ON, Canada

2 Towson University, Towson, MD, USA
This "on the fly" form of control was first demonstrated by Corballis and Gratton (2003) using a version of the typical letter flanker task (i.e., presented with a string of letters, participants had to identify a central letter while ignoring the flanking letters). In their version, the letter strings were presented equally often and unpredictably at one of three locations. Importantly however, those presented on one side of the display were more likely to be incompatible (e.g., HHSHH), while those on the other side were more likely to be compatible (e.g., HHHHH). What was found is that in the mostly incompatible (MI) location, the flanker interference effect was significantly reduced compared to the mostly compatible (MC) location. The typical explanation for this locationspecific proportion compatibility (LSPC) effect is that location serves as a contextual cue for a shift in control settings, biasing attention according to likelihood of conflict (cf., Bugg \& Crump, 2012). In addition to location-triggered adjustments of control, other features (e.g., color; Lehle \& Hubner, 2008) and categories (e.g., gender; Cañadas, Rodriguez-Bailon, \& Milliken, 2013) can serve as a cue for control as well.

Whereas LPSC effects have been known for some time, it has only recently been shown that these effects can also transfer to novel locations in space (see, e.g., Crump \& Milliken, 2009 , for transfer to novel stimuli in the same location). This transfer of LPSC effects to novel locations was first shown by Weidler and Bugg (2016), who had participants first complete 
a series of training trials in which one location (e.g., the upper right of the screen) was biased to contain mostly incompatible trials (the MI location), while another (e.g., the lower left of the screen) was biased to contain mostly compatible trials (the MC location). Training produced LSPC effects, with interference effects being smaller in the MI than the MC condition. Participants then completed a series of trials in which stimuli appeared in additional new "transfer locations" with equal numbers of compatible and incompatible trials; what was found was that LSPC effects transferred to these unbiased locations. As with the original location LSPC effects, smaller flanker compatibility effects were seen in at the location near to (i.e., spatially linked) to the MI location compared to that near the MC location.

Replicating the pattern of results found with different spatial layouts, Weidler and Bugg (2016) also found LSPC transfer to occur not only when training and transfer appeared in the same coordinate directions, but also when stimuli appeared in rings of a bulls-eye: once again smaller interference effects were seen in transfer locations in a ring trained with an MI-biased location compared to unbiased transfer locations in a ring trained with an MC-biased location. This suggests that control settings can be linked to categories of space rather than just coordinate directions. Weidler, Dey, and Bugg (2018) further demonstrated that control settings transfer to nearby locations even when a visual border is added between training and transfer locations. This transfer beyond established reference frames also occurred when training and transfer locations were made meaningfully distinct by appearing on and off an island, respectively.

While there is little doubt that LSPC effects can transfer to novel locations, it is unknown how general this transfer is. More specifically, LPSC effects have been found in a wide variety of cognitive control paradigms including the flanker task with letters (e.g., Corballis \& Gratton, 2003) or arrows (e.g., Diede \& Bugg, 2017; Weidler \& Bugg, 2016), the Simon task (Hübner \& Mishra, 2016), and variants of the Stroop task (e.g., Crump, Gong \& Milliken, 2006; Crump et al., 2008), including spatial Stroop (Funes, Lupiáñez, \& Humphreys, 2010). Given that LSPC effects occur across a range of tasks, it is reasonable to conclude that the transfer of such effects should also be generalizable. However, to date, the transfer of the LSPC effect to novel locations in space has only been demonstrated using a flanker task with arrow stimuli (Weidler \& Bugg, 2016; Weidler et al., 2018). Thus, it is not known if the transfer does indeed reflect the spatial spread of LSPC processing or something epiphenomenal to the arrow flanker task (which has a high degree of automatic response activation due to the spatial nature of the stimuli; e.g., Galfano et al., 2012). The current study was designed to examine this issue.

To determine if the location-based transfer of control settings can occur in situations beyond tasks that have strong spatial-response association (i.e., flanker with arrow stimuli), we used variations of the Stroop task. This task has the advantage of producing strong compatibility effects (e.g., MacLeod, 1991) without involving overlearned spatially directed stimuli. If the transfer of control settings to categorically related locations is a universal feature of cognitive control tasks, then LSPC effects should be found with the typical Stroop tasks. If such transfer effects are not found, this absence would highlight the fact that there are important distinctions in the mechanisms of initially acquiring and transferring conflict biases.

\section{Experiment 1}

This experiment is a conceptual replication of Weidler et al. (2018) with the important change to address the generalizability of location-specific transfer: Conflict is elicited via a Stroop task instead of an arrow flanker task. In this Stroop paradigm participants indicate the color of the ink, and conflict arises from the necessary suppression of the relatively automatic process of word reading on incompatible trials (e.g., when the stimulus BLUE is red in color; MacLeod, 1991).

\section{Method}

Participants Thirty-one undergraduates (age 18-22 years, 19 female and 12 male) participated for partial course credit. All had normal or corrected-to-normal vision and normal color vision. One participant was removed for poor performance (only $63.7 \%$ trials usable after reaction time (RT) and accuracy trim, $<3 S D$ below average proportion of usable trials, $92.1 \%$ ), resulting in a sample size of 30 .

Stimuli An LCD display (resolution $2,560 \times 1,440$ ) was viewed binocularly from approximately $57 \mathrm{~cm}$. Stimuli were the word "BLUE," "GREEN," "RED," or "YELLOW" printed in one of those four colors. In compatible trials the meaning of the word matched its physical color (e.g., "RED" written in red), whereas in incompatible trials it did not (e.g., "RED" written in blue).

Stimuli appeared one at a time at equidistant locations along a positive linear function (see Fig. 1): at one of three central locations during practice and training, and at one of five locations during transfer blocks. Locations were $5.1^{\circ}$ down and $9^{\circ}$ to the left of fixation, $10.2^{\circ}$ down and $18^{\circ}$ to the left of fixation, at fixation, and at mirror locations to the upper right. The two locations immediately to the upper right and lower left of center were biased such that one was mostly compatible (MC; $75 \%$ compatible trials) while the other was mostly incompatible (MI; 25\% compatible trials). The locations of MC and MI conditions were counterbalanced across participants. 


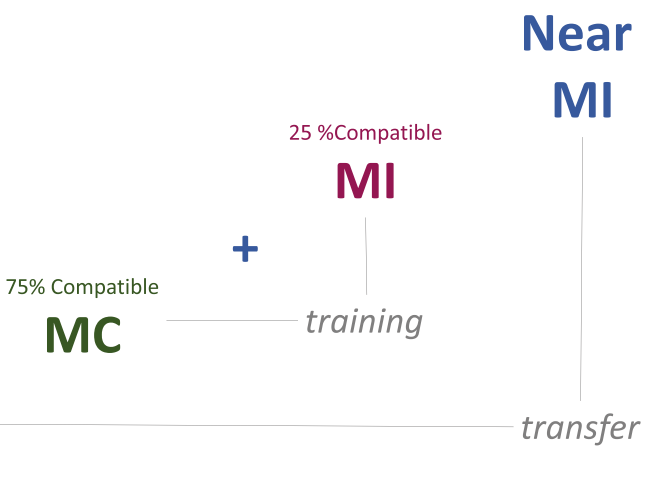

ig. 1 Depiction of the method. During all blocks there were also centrally presented items ( $50 \%$ compatible, not depicted in figure). During the training blocks items appeared centrally and at the MC ( $75 \%$ compatible) and MI (25\% compatible) locations. During Transfer blocks included stimuli appeared at all five locations. Stimuli appeared one at a time. MC and MI locations were counterbalanced across participants. Border represents computer monitor

Design and procedure On each trial a white $1^{\circ}$ fixation cross (+) followed by a $1^{\circ}$ high color word appeared on a black background. Participants responded to the physical color of the word using their right index finger on the number pad ("2" for blue, "4" for red, "6" for green, "8" for yellow).

Participants completed two practice blocks during which they received feedback on incorrect responses. This was followed by three training and two transfer blocks. Each trial began with a $1^{\circ}$ high white fixation cross at the center of the screen presented for 1,000 ms. This was followed by a word appearing at one of three locations (in practice and training) or one of five locations (in transfer) as indicated above. Stimuli were randomly chosen without replacement and remained visible until the participant made a valid response (by pressing the $2,4,6$, or 8 key on the number pad).

The first practice block consisted of 12 trials with "XXXXX" appearing at fixation in one of four colors (3 repetitions $\times 4$ colors). The second consisted of 24 trials pulled at random from the Training block. Training blocks consisted of 144 trials (48 in each of three locations). In the MC location, each of the four compatible stimuli appeared nine times (36 total), and each of the 12 incompatible stimuli appeared once (12 total). These frequencies were reversed for a total of 12 compatible and 36 incompatible trials in the MI location (each unique compatible and incompatible stimulus appeared three times). In the center, compatible and incompatible stimuli appeared with equal frequency ( 24 each).

Following training, transfer blocks of 240 trials were presented (48 trials in each of five locations). The three central locations from training retained their respective biases. The additional outer locations were the unbiased (50\% compatible) transfer locations, referred to by their proximity to trained biased MC and MI locations as near MC and near MI, respectively.

\section{Results}

Trials with response times of less than $200 \mathrm{~ms}$ or greater than $2,000 \mathrm{~ms}$ ( $2.4 \%$ of trials) were excluded from analysis and incorrect trials $(4.6 \%$ of the remaining trials) were excluded from the RT analysis. RTs for the biased items were collapsed across the five test blocks. The center location (unbiased) was not analyzed (cf., Weidler \& Bugg, 2016).

LSPC effects The RT data were analyzed with a 2 (PC: MC or $\mathrm{MI}) \times 2$ (compatibility: compatible or incompatible) repeatedmeasures analysis of variance (ANOVA). There was a Stroop effect: compatible trials $(M=768 \mathrm{~ms})$ were faster than incompatible trials $(M=827 \mathrm{~ms}), F(1,29)=57.68, p<.001, \eta_{2}{ }^{\mathrm{p}}=$ .67. There was no main effect of PC, $F(1,29)=3.73, p=.063$. PC interacted with compatibility, $F(1,29)=5.69, p=.024, \mathrm{n}_{2}{ }^{\mathrm{p}}$ $=.16$, because as typically found in LSPC paradigms, there was reduced Stroop in the MI location (46 ms) compared to the MC location (71 ms).

The same analysis conducted on error rate revealed no significant effect of PC $F(1,29)=2.25, p=.14$, nor compatibility $F(1,29)=2.27, p=.14$, and no interaction between the two $F<1, p=.36$.

Transfer effects A 2 (location: near MC or near MI) $\times 2$ (compatibility: compatible or incompatible) ANOVA also revealed a main effect of compatibility $F(1,29)=9.25 p=.005, \eta_{2}{ }^{\mathrm{p}}=$ .24 , with faster RTs in the compatible $(M=760 \mathrm{~ms})$ than incompatible $(M=822 \mathrm{~ms})$ trials. There was no effect of location, $F(1,29)=1.96, p=.172$. Importantly, there was no interaction effect, $F<1$, indicating there was no transfer of the LSPC (the compatibility effects for MC and MI locations were $32 \mathrm{~ms}$ and $38 \mathrm{~ms}$, respectively).

The same analysis conducted on error rate revealed no significant effect of PC nor compatibility $F \mathrm{~s}<1$, and no interaction between the two $F(1,29)=2.87, p=.10$. Figure 2 .

\section{Discussion}

To the best of our knowledge, this is the first experiment to examine if location-based control settings transfer to novel locations when a task other than arrow flanker is employed (e.g., Weidler et al., 2018). Two main findings emerge from this experiment. First, typical LSPC effects were seen with the Stroop task at the biased training locations. We note that this is also the first demonstration of location-specific control with "intact" Stroop stimuli (i.e., response relevant color information appearing in the word; other researchers employing the Stroop task have presented the word and color patch independently in space and time; Crump et al., 2006, Crump et al., 2008, Crump \& Milliken, 2009; Crump et al., 2018). Secondly, there was no evidence of the transfer of these effects to nearby novel locations as had been found in 

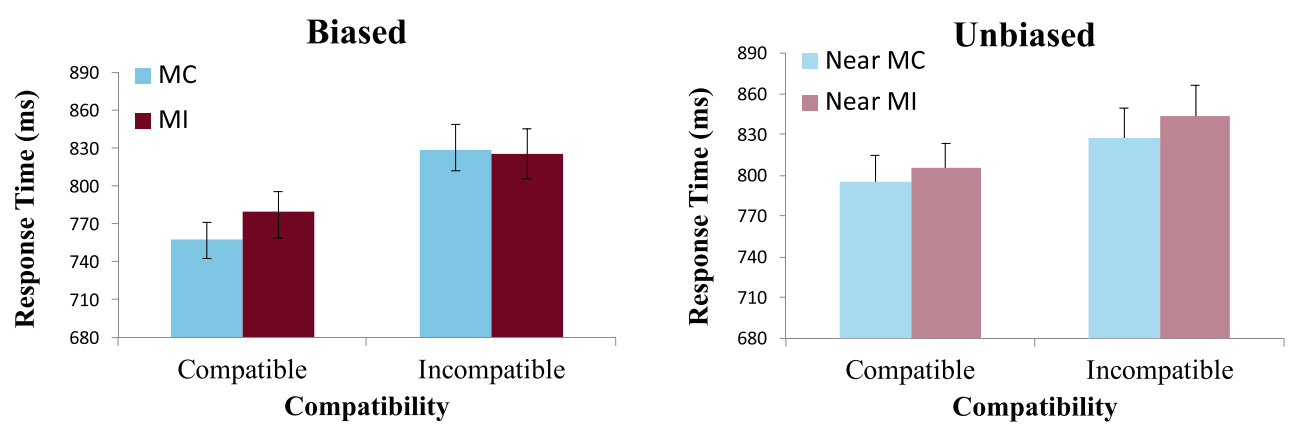

Fig. 2 Results from Experiment 1 biased training locations (left panel) and unbiased transfer locations (right panel). Error bars represent standard errors of the mean.

previous studies employing arrow flankers (Weidler \& Bugg, 2016; Weidler et al., 2018).

That LSPC effects did not transfer when employing the Stroop task suggests the difference in type of conflict elicited by the Stroop task and the arrow flanker task may modulate transfer of location-based control. Specifically, perhaps the LSPC fails to transfer to nearby unbiased locations in the Stroop task because the task requires no processing of spatial information: A single stimulus appears, and attention need only be devoted to the overall color of the word. This is in contrast to the flanker task where attention to flanking symbols must be inhibited to focus spatial attention on the central symbol in the string (e.g., Cepeda et al., 1998). To examine if spatially selecting target information from distractor information is necessary for transfer, we separated the target and distractor information in the Stroop task in Experiment 2.

\section{Experiment 2}

Experiment 2 again required participants to identify the color of stimulus while supressing meaning information, but now the distractor word and target color patch were segregated in space (e.g., Spieler, Balota, \& Faust, 2000). If what is necessary to facilitate spatial transfer of control is forcing spatial selection to complete the task (as is required to isolate the central target in a string of symbols in a flanker task in past research, e.g., Weidler et al., 2018) then there should be evidence for transfer in the present experiment.

\section{Method}

Participants Thirty undergraduate students (age 17-20 years, 22 female and eight male) participated for partial course credit. All had normal or corrected-to-normal vision and normal color vision. One participant was removed from the analysis because they did not complete the session.

Stimuli, design, and procedure The method was as in Experiment 1 except that segregated Stroop stimuli were used in place of integrated. The color word (RED, YELLOW, GREEN, or BLUE) written in white appeared simultaneously with a $4^{\circ} \times 1^{\circ}$ colored rectangle (see Fig. 3 ). The rectangle and word were either compatible (e.g., red with "RED") or incompatible (e.g., red with "BLUE"). The word was randomly selected to appear above or below the rectangle in each trial.

\section{Results}

Trials with RTs of less than $200 \mathrm{~ms}$ or greater than 2,000 ms (3.9\% of trials) were excluded from analysis and incorrect trials (5.4\% of the remaining trials) were excluded from the RT analysis.

LSPC effects The 2 (PC: MC or MI) $\times 2$ (compatibility: compatible or incompatible) repeated-measures ANOVA on RT revealed that participants responded significantly faster to compatible compared to incompatible stimuli $F(1,28)=20.76, p<.001, \eta_{2}{ }^{\mathrm{p}}=.43$. There was no main effect of PC $(F<1)$, but PC interacted significantly with compatibility, $F(1,28)=5.96, p=.021, \eta 2^{\mathrm{p}}=.18$. Demonstrating the LSPC effect, compatibility effects were smaller in the MI location $(M=21 \mathrm{~ms})$ compared to the $\mathrm{MC}$ location $(M=55 \mathrm{~ms})$.

The same analysis conducted on error rate revealed no significant effect of PC or compatibility, $F \mathrm{~S}<1$, and no interaction between the two $F(1,28)=1.03, p=.32$.

Transfer effects The 2 location (near MC or near MI) $\times 2$ (compatible or incompatible) ANOVA revealed that Stroop effects were intact, $F(1,28)=5.99, p=.021, \eta_{2}{ }^{\mathrm{p}}=.18$, with faster RTs in the compatible $(M=762 \mathrm{~ms})$ than incompatible $(M=796 \mathrm{~ms})$ trials. There was no effect of location $(F<1)$. Furthermore, in the transfer items there was no interaction of location with compatibility, $F<1$, indicating no transfer of LSPC effects. The compatibility effects were equivalent in the two locations, with $M=22 \mathrm{~ms}$ and $M=24 \mathrm{~ms}$ in MC and MI, respectively. The same analysis conducted on error rate revealed no significant effect (all $F s<1$ ). Figure 4. 


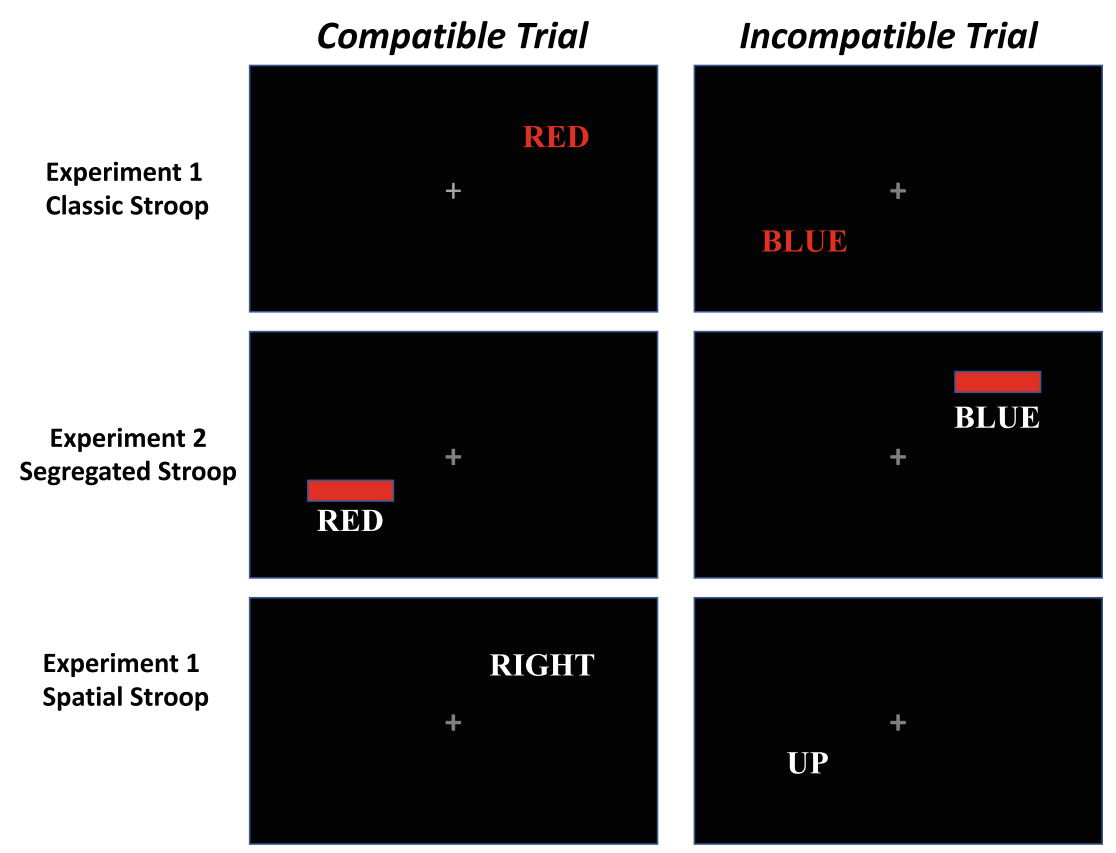

Fig. 3 Example compatible and incompatible trial for each experiment. Not depicted to scale

\section{Discussion}

This experiment differed from Experiment 1 in that it required spatial selection between two simultaneously appearing elements of segregated Stroop stimuli. As in the first experiment, LSPC effects were seen at biased training locations but did not transfer to novel unbiased locations. This suggests that forcing spatial selection is not sufficient to elicit the spatial transfer of control. Of course, the arrow flanker task in which transfer has been demonstrated in prior research does not merely force spatial selection, but additionally the irrelevant information that must be suppressed (the direction of the flanking arrows) is inherently spatial in nature. By contrast, in both of the Stroop tasks employed in Experiment 1 and Experiment 2, the conflict arises from suppressing automatic meaning processing to respond to a physical color (DeSoto et al., 2001). In Experiment 3, we tested the hypothesis that spatially driven conflict is necessary for spatial transfer of control.

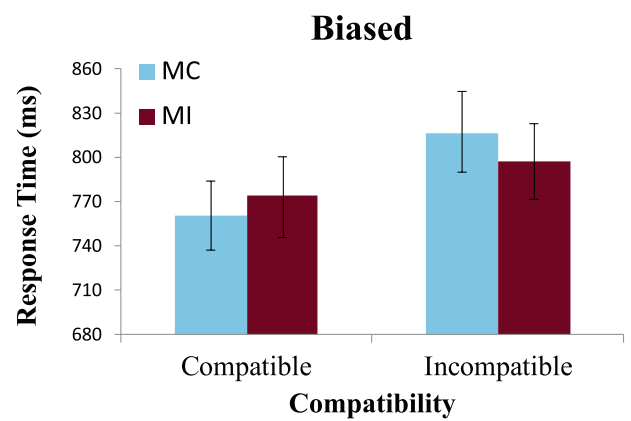

\section{Experiment 3}

From the previous two experiments it appears not every conflict task (Experiment 1) or every conflict task that that requires spatial selection between two stimuli (Experiment 2) will facilitate spatial transfer of control. It may be that transfer effects only occur in situations where the conflict has a strong spatial component as in the arrow flanker tasks. In order to test this hypothesis within the Stroop paradigm, the present experiment employs the spatial Stroop task. This task causes conflict between the meaning of a location word and its physical location in space (e.g., Lu \& Proctor, 1995). The spatial Stroop task is in this way similar to the arrow flanker task, in that resolution of conflict requires attention to task-relevant spatial information and controlled exclusion of irrelevant spatial information. If it is this controlled allocation of attention to spatial dimensions that facilitates the learning of spatial categories within which control settings can transfer, we would expect to see strong a LSPC transfer effect in the spatial Stroop task.

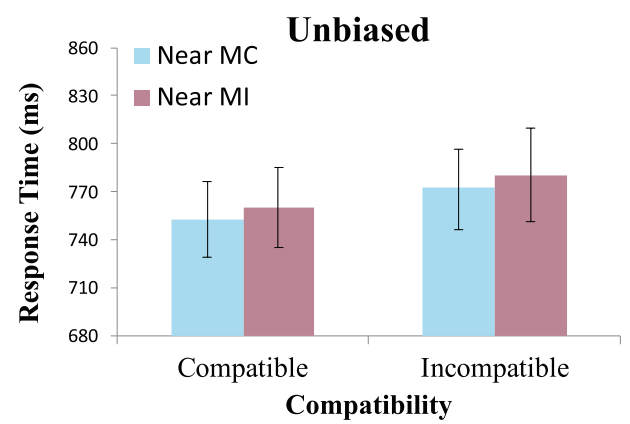

Fig. 4 Results of Experiment 2 biased training locations (left panel) and unbiased transfer (right) using segregated Stroop stimuli. Error bars represent standard errors of the mean 


\section{Method}

Participants Thirty-three undergraduate students (age 17-30 years, 23 female and ten male) participated for partial course credit. All had normal or corrected-to-normal vision.

Stimuli, design, and procedure The method was as in Experiment 1 except for the following. On each trial one of four direction words (up, down, left, or right) appeared in white capital letters. Participants responded to the meaning of the word by pressing the corresponding arrow key on the number pad with their right index finger (e.g., "4" for LEFT).

For each location there were two possible compatible (e.g., LEFT or DOWN when the stimuli were to the left of fixation) and two possible incompatible stimuli (e.g., RIGHT or UP in that same location). In the MC location each of two compatible stimuli appeared 18 times (36 total), and each of two incompatible stimuli appeared six times (12 total). In the MI location this was reversed, with 36 total incompatible and 12 total compatible stimuli appearing. In the center and transfer locations each word appeared 12 times (which resulted in 24 compatible and 24 incompatible trials in transfer locations; compatibility is not applicable in the central location in this Experiment). Total trial numbers were the same as in Experiment 1. Practice trials, during which feedback was given for incorrect responses, consisted of 12 trials of stimuli appearing at fixation followed by 12 trials identical to training with stimuli at one of three central locations.

\section{Results}

Trials with response times of less than $200 \mathrm{~ms}$ or greater than $2,000 \mathrm{~ms}(.5 \%$ of trials) were excluded from analysis and incorrect trials (2.4\% of the remaining trials) were excluded from the RT analysis.

LSPC effects The 2 (PC: MC or MI) $\times 2$ (compatibility: compatible or incompatible) repeated-measures ANOVA revealed a spatial Stroop effect: Compatible trials $(M=671 \mathrm{~ms})$ were faster than incompatible trials $(M=706 \mathrm{~ms}), F(1,32)=$ $91.12, p<.001, \eta_{2}{ }^{\mathrm{p}}=.74$. There was no main effect of PC, $F(1,32)=2.87, p=.100$. PC interacted significantly with compatibility, $F(1,32)=68.44, p<.001, \eta 2^{\mathrm{p}}=.68$ because the Stroop effect was larger in the MC location $(91 \mathrm{~ms})$ than in the MI location (-22 ms).

The same analysis conducted on error rate mirrored the RT pattern: Compatible trials $(M=1.87 \%)$ has a significantly lower error rate than incompatible trials $(M=3.11 \%), F(1$, $32)=8.68, p=.006, \eta_{2}{ }^{\mathrm{p}}=.21$. There was no effect of location $F(1,32)=1.23, p=.28$. PC interacted significantly with compatibility, $F(1,32)=9.75, p=.004, \eta_{2}{ }^{\mathrm{p}}=.23$, with the increased error rate for incompatible trials being larger in the MC $(2.55 \%)$ than in the MI (-.05\%) location.
Transfer effects The 2 (location: near MC or near MI) $\times 2$ (compatibility: compatible or incompatible) ANOVA also revealed that compatible trials $(M=736 \mathrm{~ms})$ were faster than incompatible trials $(M=759 \mathrm{~ms}), F(1,32)=15.63 p<.001$, $\eta_{2}{ }^{\mathrm{p}}=.33$. There was no effect of location, $F<1$. Importantly, location and compatibility interacted, $F(1,32)=17.55 p<$ $.001, \eta_{2}{ }^{p}=.35$. This interaction mirrored the typical LSPC effect and that found in prior work investigating transfer: compatibility effects were smaller for $50 \%$ compatible items appearing near the MI items $(-17 \mathrm{~ms})$ than those appearing near the MC items (64 ms).

The same analysis conducted on error rate revealed similar results: Compatible trials $(M=5.97 \%)$ has a significantly lower error rate than incompatible trials $(M=6.42 \%), F(1,32)=$ $15.27, p<.001, \mathrm{\eta}_{2}{ }^{\mathrm{p}}=.32$. There was no effect of $\mathrm{PC} F(1,32)$ $<1, p=.59$. PC interacted significantly with compatibility, $F(1,32)=4.56, p=.040, \eta_{2}{ }^{\mathrm{p}}=.13$, with a greater increase in error rate for incompatible trials in the $\mathrm{MC}(2.55 \%)$ than in the MI (.74\%) location. Figure 5.

\section{Discussion}

In the present experiment, we demonstrate transfer of location-specific control to novel locations in a task other than the arrow flanker task used in prior research. In this spatial Stroop task, participants had to engage control to suppress the irrelevant location in which the stimulus appeared (e.g., DeSoto et al., 2001). Both LSPC effects and transfer effects were observed, supporting the hypothesis that resolving spatial conflict is a key feature of cognitive control tasks that produce transfer to new locations in space. We note that in this experiment both the training and transfer effects are in part driven by a reversal of the compatibility effect in the MI condition. This is, however, not an uncommon pattern in the spatial Stroop task (see, e.g., Logan \& Zbrodoff, 1979, for a similar reversal in a PC manipulation).

\section{Additional analyses}

For a more thorough evaluation of our data across experiments, we performed three additional analyses. First, given the similarity of the design of experiments, we repeated the main training and transfer analyses including experiment as a between-subjects factor. Second, we examined how the LSPC in the biased items potentially differed between the training blocks (earlier in the experiment, when there were only three possible locations) and transfer blocks (later in the experiment, when unbiased items also appeared for a total of five possible locations). Finally, we report compatibility effects for the center location (for Experiments 1 and 2, as this is not applicable in Experiment 3), also as a function of training and transfer. 

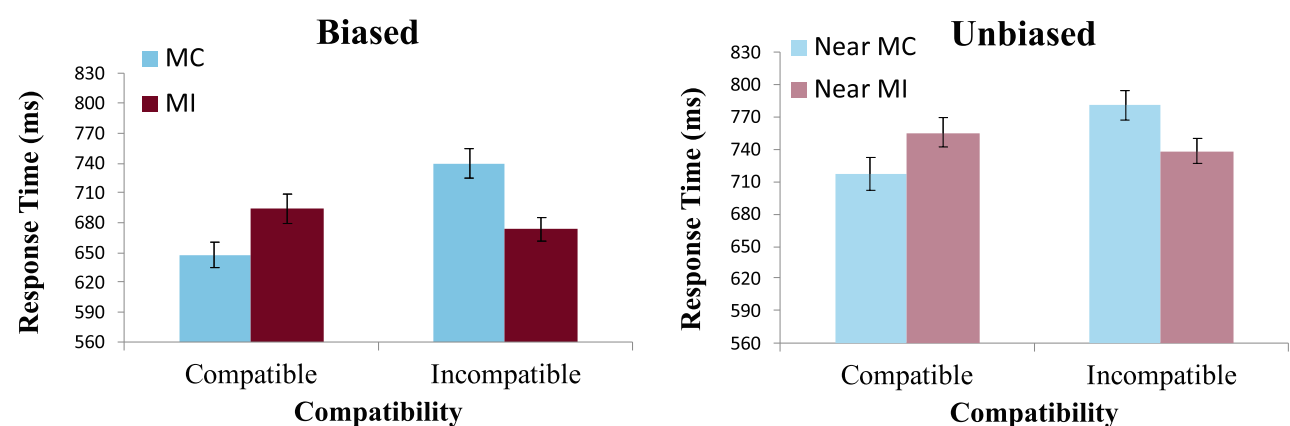

Fig. 5 Results of Experiment 3 biased training locations (left panel) and unbiased transfer (right) using spatial Stroop stimuli. Error bars represent standard errors of the mean

Between-experiment analyses For biased items, a 3 Experiment $\times 2 \mathrm{PC} \times 2$ compatibility mixed ANOVA with Experiment as the between subjects factor revealed an effect of Experiment, $F(2,89)=10.41, p<.001, \eta_{2}{ }^{\mathrm{p}}=.19$. Bonferroni-corrected pairwise comparisons revealed RTs overall were faster in Experiment $3(688 \mathrm{~ms})$ than in either Experiment 1 (798 ms; $p<.001)$ or Experiment $2(787 \mathrm{~ms} ; p=$ $.001)$. Experiment interacted with compatibility, $F(2,89)=$ $3.69, p=.029, \eta_{2}{ }^{\mathrm{p}}=.08$ (compatibility effects larger in Experiment 1, $59 \mathrm{~ms}$, than in Experiments 2, $39 \mathrm{~ms}$ or 3, 34 $\mathrm{ms})$. Experiment also marginally interacted with PC, $F(2,89)$ $=2.68, p=.074,{ }_{\eta_{2}}{ }^{\mathrm{p}}=.06$ (RTs were $10 \mathrm{~ms}$ faster in $\mathrm{MC}$ than $\mathrm{MI}$ in Experiment 1, but $2 \mathrm{~ms}$ and $9 \mathrm{~ms}$ faster $\mathrm{MI}$ in Experiments 2 and 3, respectively). In addition there was a three-way interaction, $F(2,89)=15.22, p<.001, \eta_{2}{ }^{\mathrm{p}}=.26$, as LSPC effects were larger in Experiment 3 (113 ms) than in Experiment 1 (25 ms) or Experiment 2 (34 ms).

The same analysis on transfer items also revealed a main effect of Experiment, $F(2,89)=10.41, p<.001, \eta_{2}{ }^{\mathrm{p}}=.19$, although for the transfer items the only reliable RT difference was between Experiment 1 (818 ms) and Experiment 3 (748 $\mathrm{ms} ; p=.031$; RTs in Experiment $2=766 \mathrm{~ms}$ ). The only effect of Experiment was its involvement in the three way interaction, $F(2,89)=8.07, p=.001,{ }_{\eta}{ }^{p}=.15$, because the $\mathrm{PC} \times$ trial type interaction selectively arose in Experiment 3 (81 $\mathrm{ms}$ and 6 and $-2 \mathrm{~ms}$ in Experiments 1 and 2, respectively).

Biased items in training versus transfer To examine the LSPC effect for biased items as a function of the training or transfer phase we conducted a 2 phase (training vs. transfer) $\times 2 \mathrm{PC} \times$ 2 compatibility repeated-measures ANOVA on each experiment. In Experiment 1 there was an effect of phase, $F(1,29)=$ $10.10, p=.004, \eta_{2}{ }^{\mathrm{p}}=.26$, with slower RTs during training (808) than transfer (782), and a marginal trial type by phase interaction, $F(1,29)=3.50, p=.071, \eta_{2}{ }^{\mathrm{p}}=.11$, because compatibility effects were larger during training $(67 \mathrm{~ms})$ than transfer $(48 \mathrm{~ms})$. Phase did not modulate the $\mathrm{PC} \times$ trial type interaction, $F<1$. In the same analysis on Experiment 2, RTs were again faster for biased items in the transfer (752) than training (809) trials, $F(1,28)=35.20, p<.001, \eta_{2}{ }^{\mathrm{p}}=.56$.
Phase again marginally modulated the compatibility effect, $F(1,28)=3.16, p=.087,{ }_{\eta_{2}}{ }^{\mathrm{p}}=.10$; however in Experiment 2 the compatibility effects were larger during transfer (53 ms) than training $(30 \mathrm{~ms})$. Phase again did not modulate the $\mathrm{PC} \times$ trial type interaction, $F(1,28)=2.08 p=.16,{ }_{\eta 2}{ }^{\mathrm{p}}=.07$.

The analyses in Experiment 3 revealed no main effect of phase, $F<1$. Phase did interact with compatibility, $F(1,32)=$ $16.47, p<.001,{ }_{\eta_{2}}{ }^{\mathrm{p}}=.34$, because compatibility effects were larger during training $(44 \mathrm{~ms})$ than transfer $(21 \mathrm{~ms})$. Phase also modulated the $\mathrm{PC} \times$ trial type interaction, $F(1,32)=6.95, p=$ $.013, \eta_{2}{ }^{\mathrm{p}}=.18$, because the LSPC effect similarly was larger during training $(127 \mathrm{~ms})$ than transfer $(90 \mathrm{~ms})$.

Compatibility effects in center location In addition, we report compatibility effects for the unbiased center location, as a function of training versus transfer blocks. In Experiment 1 the only statistically significant effect from a 2 phase $\times 2$ trial type ANOVA was that of compatibility, $F(1,29)=50.35, p<$ $.001, \eta_{2}{ }^{p}=.64$ (average Stroop interference was $84 \mathrm{~ms}$ at the center unbiased location). The same analysis on Experiment 2 again revealed an effect of compatibility, $F(1,28)=16.29, p<$ $.001, \eta_{2}{ }^{p}=.37$ (average Stroop effect was $35 \mathrm{~ms}$ ). There was also a main effect of phase, $F(1,28)=22.50, p<.001, \eta_{2}{ }^{\mathrm{p}}=$ .45 , with faster RTs at the center location during transfer (734 $\mathrm{ms})$ than training $(783 \mathrm{~ms})$. The factors did not interact in either experiment, $F_{\mathrm{S}}<1$. (Note that we do not report compatibility effects for Experiment 3 as in the center location trials cannot be compatible or incompatible.)

\section{General discussion}

The present investigation explored the generalizability of the transfer of LSPC effects to novel unbiased locations. As the only previous work demonstrating transfer had done so with arrow flanker tasks, we tested three variations of the Stroop task. The LSPC for biased items was observed in all variations of the Stroop task tested. The same was not true for LSPC transfer, as the first two experiments provided no evidence for transfer in color Stroop tasks, regardless of whether stimuli 
were integrated (a color word written in a certain color; Experiment 1) or spatially segregated (a color word appearing in white above or below a colored rectangle; Experiment 2). In contrast, the spatial Stroop task (Experiment 3) did result in strong transfer effects.

These results imply that, while location-specific bias of control settings occurs across a variety of conflict tasks, the transfer of control settings within categories of space is more restricted. The necessary feature for transfer appears to be that spatial information is involved in the conflict. In neither of the color Stroop tasks, but in both the arrow flanker (in prior research) and spatial Stroop tasks (in the present research), conflict arises between spatial stimulus dimensions (conveyed by the direction of arrows or a direction word, respectively). Though segregated color Stroop required spatial selection of the relevant stimulus dimension, this spatial dimension was not involved in the conflict (which like in the classic Stroop arose between word meaning and physical color). It is therefore not sufficient that a task involves spatial selection; the spatial information must be a source of conflict to facilitate learning of spatial categories within which control settings transfer.

Before fully moving ahead with the conclusion that is was the spatial conflict in the third experiment that finally generated the transfer effects, a few other alternatives should be considered. Perhaps the difference in task demands from the color identification tasks of the first two experiments to the word identification task in the final experiment produced the different patterns? This seems unlikely as the overall transfer RTs from Experiment 2 are statistically equivalent to that in Experiment 3, suggesting that the tasks were not all that different in difficulty. Perhaps is was simply the different discrimination tasks (color vs. word) themselves, aside from overall difficulty, that produced the different pattern of results? However, given the comparable qualitative pattern in the training trials across tasks, it isn't clear why the color task aside from not having a spatial conflict component - would selectively not generate transfer effects. Another consideration is that the typical LSPC paradigm we adapted for this study does have the transfer stimuli presented further away from fixation, and this might have made the color identification tasks relatively easier (i.e., easier to ignore the words) and the word identification task more difficult. However, as noted before, the transfer RTs were comparable between Experiments 2 and 3. Also, it is important to remember that the two training and the two transfer locations in each experiment were equidistant from fixation, therefore any difficulties that theoretically might arise (in either word reading or color processing) would be equivalent in the conditions of critical comparison. Finally, differences in experimental layout may have played a roll; in the spatial Stroop task of Experiment 3, each specific location (e.g., lower left) could only have two possible compatible responses (e.g., left and down) and two possible incompatible responses (e.g., right and up), whereas in the color Stroop task all four possible responses could be compatible and incompatible in each location. However, the arrow flanker tasks that have produced location-based transfer of LSPC effects in the past (e.g., Weidler et al., 2018) also afford all four responses to be incompatible/compatible in each location, making it unlikely that this difference is driving the present results. Overall, the finding of LSPC transfer effects only in the spatial Stroop experiment is most parsimoniously accounted for by existence of spatial conflict in that task.

That spatial transfer is only observed in tasks involving spatial conflict is consistent with a memory-based mechanism of LSPC effects (Chui \& Egner, 2019). In this view, contextual cues indicative of conflict are encoded with the associated attentional control state, permitting rapid retrieval of the appropriate control upon stimulus onset in a given context. This account is supported by neural data illustrating that "on the fly" context-specific activity in sensory processing regions coupled to apparent top-down control from frontal regions predicts the behavioral LSPC effects (King, Korb \& Egner, 2012). Thus, LSPC effects in this framework are the result of episodic event representations that bind top-down control settings with implicit contextual cues, so that stimuli appearing in a location associated with high conflict for example will "onthe-fly" reinstate this control set.

Importantly, not all contextual information present in a situation producing conflict can become a cue for future adaptive control. Only certain features of the context become episodically bound (Surrey et al., 2017), and there is evidence that selectively attended features are preferentially encoded. The hippocampus is well known for its role in spatial learning and episodic binding, and its activity during encoding is predictive of subsequent benefits in rapid implicit cueing of attention by contextual features (Goldfarb, Chun, \& Phelps, 2016). Furthermore, this hippocampal activity occurs in dissociable regions during encoding and retrieval of location and color cues (Uncapher \& Rugg, 2009). Importantly, this dissociable activity is observed during the online processing of color or location independently predicts future memory specifically for those contextual features (Uncapher, Otten, \& Rugg, 2006). Together these findings imply that a task directing selective attention towards a certain feature (e.g., location or color) would increase the potency of that feature serving as an episodic cue for the retrieval of control settings.

Tasks such as the spatial Stroop and arrow flanker make space a relevant contextual feature by requiring the inhibition of automatic spatial orienting. Thus, one might expect this to lead to broader encoding of space. When stimuli subsequently appear in new locations within the spatial category of a learned conflict bias, the associated control set is cued for retrieval and the LSPC effect would be expected to transfer, as it did in Experiment 3. On the other hand, the contextual features that must be inhibited in the color Stroop task do not direct attention to space, therefore one might expect that 
location will be encoded much more narrowly (i.e., only where the biased items appear). In this case, when stimuli appear in novel nearby locations they do not trigger retrieval of the learned control settings and the LSPC effects do not transfer (as in Experiments 1 and 2). An interesting avenue for future research is to examine how making different features task relevant might facilitate transfer along different dimensions.

The major theoretical implication that arises from the present study concerns the conceptualization of how cognitive control processes facilitate learning about regularities in the environment (e.g., how a certain location predicts conflict). Specifically, the processes underlying non-spatial-based and spatial-based conflict tasks appear to produce dissociable learning effects, but this dissociation is only visible when examining transfer to conflict-unbiased items. Prior to the present findings, it is safe to say that the conflict processes that produced LSPC effects were considered fungible; at this resolution, any conflict would create differences at MC and MI locations. However, the present findings indicate that LSPC transfer effects are specific to tasks with spatial conflict. The LSPC effects produced by non-spatial conflicts conversely are bound strictly to the spatial locations at which they occur, making them indeed locationspecific proportion compatibility effects.

To summarize, these experiments specify the conditions under which LSPC effects can transfer to novel unbiased locations. Though a number of conflict tasks produce reliable LSPC effects, a smaller set of these tasks also produce transfer. The transfer of control settings relies on learning spatial categories, which is facilitated by tasks whose source of conflict is between spatial dimensions. Further studies employing other cognitive control tasks that have shown LSPC effects may help to further clarify the necessary features of conflict that result in spatially transferable control of attention.

\section{References}

Bugg, J. M., \& Crump, M. J. (2012). In support of a distinction between voluntary and stimulus-driven control: A review of the literature on proportion congruent effects. Frontiers in psychology, 3, 367.

Cañadas, E., Rodríguez-Bailón, R., Milliken, B., \& Lupiáñez, J. (2013). Social categories as a context for the allocation of attentional control. Journal of Experimental Psychology: General, 142(3), 934.

Cepeda, N. J., Cave, K. R., Bichot, N. P., \& Kim, M. S. (1998). Spatial selection via feature-driven inhibition of distractor locations. Perception \& Psychophysics, 60(5), 727-746.

Chiu, Y. C., \& Egner, T. (2019). Cortical and subcortical contributions to context-control learning. Neuroscience \& Biobehavioral Reviews.

Cohen, J. (2017). Cognitive Control: Core Constructs and Current Considerations. In T. Egner (Ed.), The Wiley Handbook of Cognitive Control (pp. 3-28). Wiley.

Corballis, P. M. \& Gratton, G. (2003). Independent control of processing strategies for different locations in the visual field. Biological Psychology, 64, 191-209.
Crump, M., Vaquero, J. M., \& Milliken, B. (2008). Context-specific learning and control: The roles of awareness, task relevance, and relative salience. Consciousness and Cognition, 17, 22-36. https:// doi.org/10.1016/j.concog.2007.01.004

Diede, N. T., \& Bugg, J. M. (2017). Cognitive effort is modulated outside of the explicit awareness of conflict frequency: Evidence from pupillometry. Journal of Experimental Psychology: Learning, Memory, and Cognition, 43(5), 824.

Crump, M. J., Gong, Z., \& Milliken, B. (2006). The context-specific proportion congruent Stroop effect: Location as a contextual cue. Psychonomic bulletin \& review, 13(2), 316-321.

Crump, M. J., Milliken, B., Leboe-McGowan, J., Leboe-McGowan, L., \& Gao, X. (2018). Context-dependent control of attention capture: Evidence from proportion congruent effects. Canadian Journal of Experimental Psychology/Revue canadienne de psychologie expérimentale, 72(2), 91.

Crump, M. J. C. \& Milliken, B. (2009). The flexibility of context-specific control: Evidence for context-driven generalization of item-specific control settings, The Quarterly Journal of Experimental Psychology, 62, 1523-1532, https://doi.org/10.1080/17470210902752096

DeSoto, M. C., Fabiani, M., Geary, D. C., \& Gratton, G. (2001). When in doubt, do it both ways: brain evidence of the simultaneous activation of conflicting motor responses in a spatial stroop task. Journal of Cognitive Neuroscience, 13(4), 523-536.

Funes, M. J., Lupiáñez, J., \& Humphreys, G. (2010). Sustained vs. transient cognitive control: Evidence of a behavioral dissociation. Cognition, 114(3), 338-347.

Galfano, G., Dalmaso, M., Marzoli, D., Pavan, G., Coricelli, C., \& Castelli, L. (2012). Eye gaze cannot be ignored (but neither can arrows). The Quarterly Journal of Experimental Psychology, 65(10), 1895-1910.

Goldfarb, E. V., Chun, M. M., \& Phelps, E. A. (2016). Memory-guided attention: independent contributions of the hippocampus and striatum. Neuron, 89(2), 317-324.

Hübner, R., \& Mishra, S. (2016). Location-specific attentional control is also possible in the Simon task. Psychonomic bulletin \& review, 23(6), 1867-1872.

King, J. A., Korb, F. M., \& Egner, T. (2012). Priming of control: Implicit contextual cuing of top-down attentional set. Journal of Neuroscience, 32(24), 8192-8200.

Logan G. D., \& Zbrodoff, N. J. (1979). When it helps to be misled: Facilitative effects of increasing the frequency of conflicting stimuli in a Stroop-like task. Memory \& cognition, 7(3), 166-174.

Lowe, D. G., \& Mitterer, J. O. (1982). Selective and divided Attention in a Stroop task. Canadian Journal of Psychology/Revue canadienne de psychologie, 36(4), 684.

Lu, C. H., \& Proctor, R. W. (1995). The influence of irrelevant location information on performance: A review of the Simon and spatial Stroop effects. Psychonomic bulletin \& review, 2(2), 174-207.

MacLeod, C. M. (1991). Half a century of research on the Stroop effect: an integrative review. Psychological bulletin, 109(2), 163.

Spieler, D. H., Balota, D. A., \& Faust, M. E. (2000). Levels of selective attention revealed through analyses of response time distributions. Journal of Experimental Psychology: Human Perception and Performance, 26(2), 506.

Surrey, C., Dreisbach, G., \& Fischer, R. (2017). Context-specific adjustment of cognitive control: Transfer of adaptive control sets. The Quarterly Journal of Experimental Psychology, 70(11), 2386-2401.

Uncapher, M. R., Otten, L. J., \& Rugg, M. D. (2006). Episodic encoding is more than the sum of its parts: an fMRI investigation of multifeatural contextual encoding. Neuron, 52(3), 547-556.

Uncapher, M. R., \& Rugg, M. D. (2009). Selecting for memory? The influence of selective attention on the mnemonic binding of contextual information. Journal of Neuroscience, 29(25), 8270-8279. 
Weidler, B. J., \& Bugg, J. M. (2016). Transfer of location-specific control to untrained locations. The Quarterly Journal of Experimental Psychology, 69(11), 2202-2217.

Weidler, B. J., Dey, A., \& Bugg, J. M. (2018). Attentional control transfers beyond the reference frame. Psychological research, 1-14.
Publisher's note Springer Nature remains neutral with regard to jurisdictional claims in published maps and institutional affiliations. 